\title{
Dysplastic Nevus and BCC development after antihypertensive therapy with Valsartan and Hydrochlorothiazide!?
}

\author{
Valeri Malev ${ }^{1}$, Georgi Tchernev ${ }^{1^{*}}$ \\ ${ }^{1}$ "Onkoderma"- Policlinic for Dermatology and Dermatologic Surgery, Sofia, Bulgaria
}

\begin{abstract}
Received: December 17, 2019; Accepted: December 17, 2019; Published: December 18, 2019
*Corresponding author: Prof Dr. Georgi Tchernev, 1"Onkoderma"- Policlinic for Dermatology and Dermatologic Surgery, Sofia, Bulgaria. E-mail: georgi_tchernev@yahoo.de
\end{abstract}

Dysplastic nevi are an important factor, associated with high risk of developing cutaneous melanoma [1]. On one hand they could be a direct precursor in up to $29 \%$ of the cases of developing cutaneous melanoma [2]. On the other hand finding dysplastic nevi on the body is strongly associated with developing of de novo melanomas [3]. At the moment there is not an innovative, definitive concept, explaining the causes of dysplastic nevi's clinical manifestation, regarding their sporadic or genetically determined evolution. Drug mediated cancerogenesis is rather a myth than a reality, but at this moment we can mark that: 1) the number of studies, exploring the link between systemic medication and various cancer types, even retrospectively, is quite low, and also 2) there seems to be a certain kind of protectionism over specific pharmaceutical giants, their products and the regulations they are subject to.

Lately, it is extensively debated on the possibility of increased risk of developing different malignant neoplasms (prostate carcinoma, skin melanoma etc.) after antihypertensive medication with sartans and thiazide diuretics for example [4-6].
It is established that some angiotensin receptor blockers (like Losartan) affect the melanogenesis, reducing the melanocytes' migration activity on one hand, but drastically stimulating their adhesion and invasion on the other [7]. Therefore if ARB's could be associated with cutaneous melanoma, then it is logical that systemic medication with ARB's can be also associated with developing dysplastic nevi $[4,5,7,8]$. As a conclusion of the mentioned facts we see two possibilities: 1) cancerogenesis triggered by different cancerogenic adultearnts (NDEA, NDMA), or 2) it is triggered by directly affecting the melanogenesis not by the supplements, but by the generic itself $[7,8]$.

We present a 60 year old male patient, accepted for surgical treatment of two lesions on the back (epidermal cyst and a dysplastic nevus) (fig 1a-c;2a-c). The patient is on antihypertensive therapy with Lercanidipine $10 \mathrm{mg}$ (1-0-1) and combination Valsartan/Hydrochlorothiazide $160 \mathrm{mg} / 12,5 \mathrm{mg}$ (10 -0) since August 2013. Two years later the patient was operated for a keratinocyte tumor on the back, later classified as basal cell carcinoma.
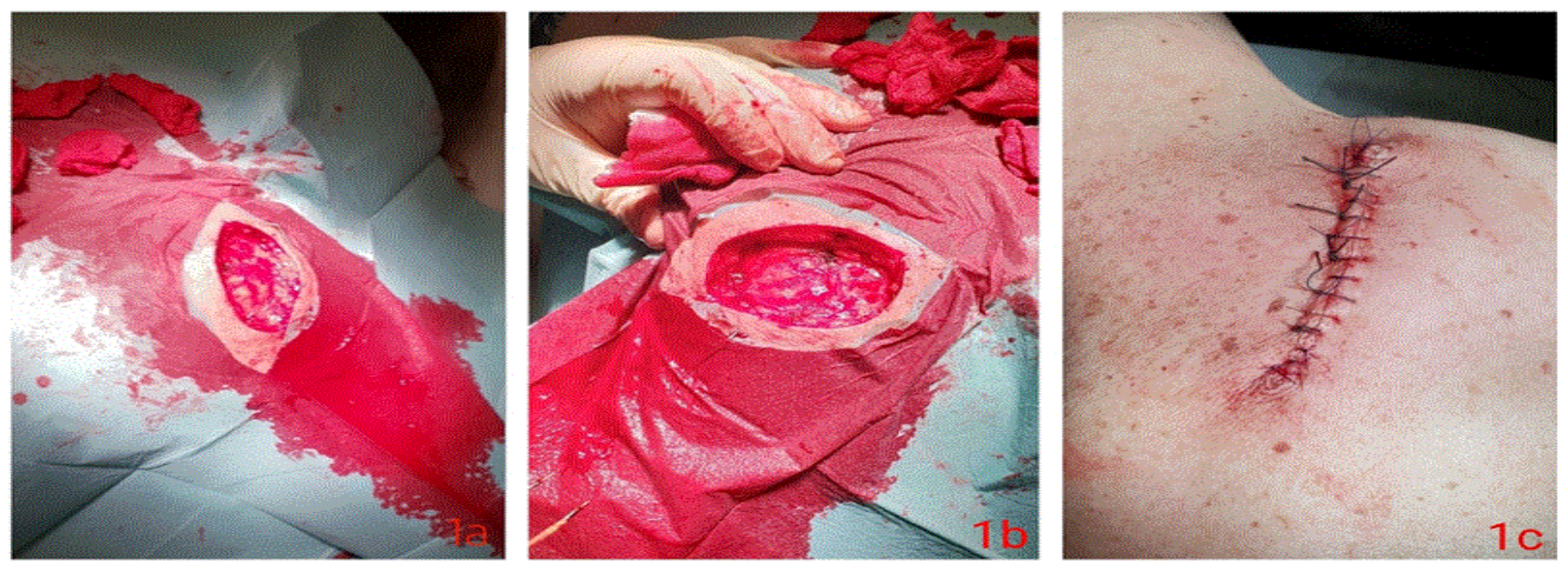

Figure: 1a-b: Intraoperative finding of a skin defect after surgical excision of epidermal cyst on the right shoulder

Figure: 1c: Postoperative finding of a closed surgical wound with single discontinued skin suture 
Before this surgical intervention (2015) the patient has been thoroughly examined clinically and via dermatoscopy (every year) and other suspicious skin lesions have not been found. According to the anamnestic data the current pigmented lesion appeared in 2016 (approximately 2,5 years after the onset of the mentioned medication) and subsequently grew rapidly and changed its color. The patient's skin is phototype II, has neither severe exposure to sunlight nor significant sunburns in childhood.

During the dermatological examination we established 2 lesions on the back. The first of them was localized on the right shoulder, $5 \mathrm{~cm}$ large, smooth, rounded, hyperemic, poorly movable, painful formation, corresponding clinically to an epidermal cyst (pic 1a-c). It was removed with a deep elliptical excision to the muscle, as the postoperative defect was closed with single, discontinued skin sutures. The histopathological diagnosis showed a ruptured keratinous epidermal cyst.

The second lesion was localized in the right subscapular area and was a nonhomogeneous pigmented lesion, $2 \mathrm{~cm}$ large, not elevated, with clear borders. The lesion was suspicious clinically and dermatoscopically for dysplastic nevus or melanoma insitu, which obliged us to remove it surgically (figs. 2a-c). The lesion was removed by a elliptical excision with surgical security margin of $1 \mathrm{~cm}$ in all directions. The defect was closed with single, discontinued skin sutures (figs. 2b-c). The histopathological diagnosis showed dysplastic junctional melanocytic nevus with clear resection lines.
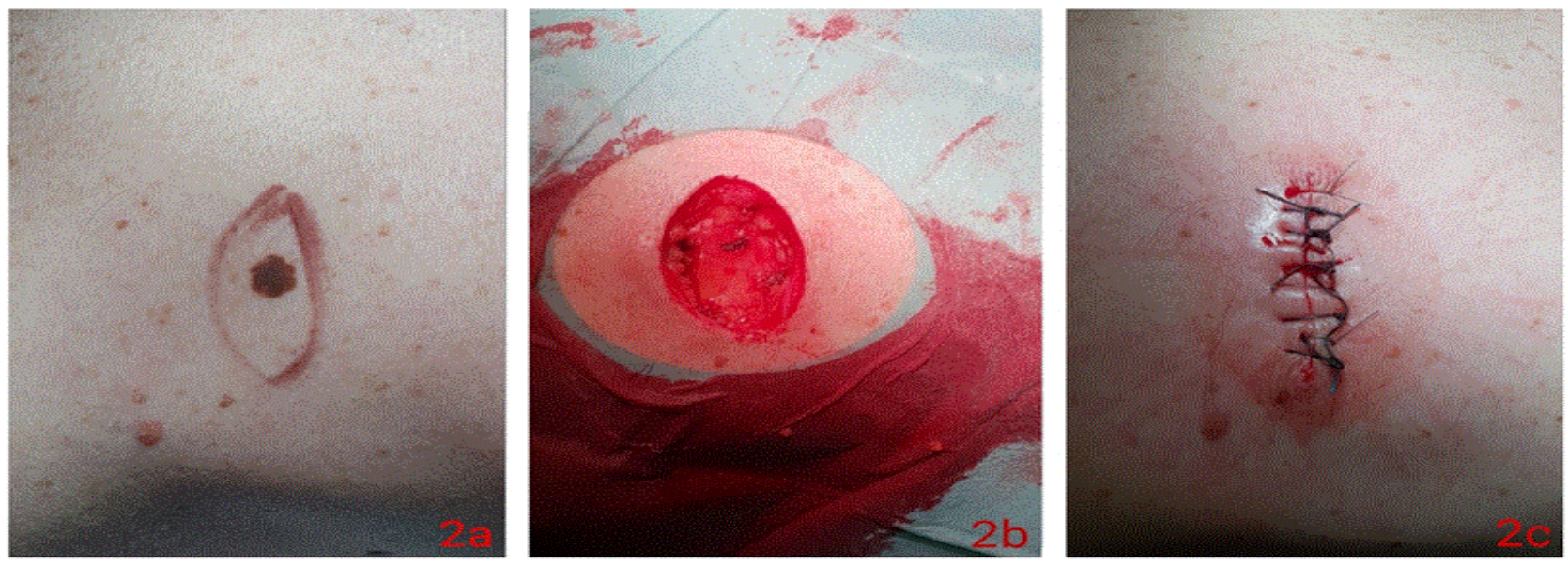

Figure: 2a: Pigmented lesion in right subscapular area

Figure: $\mathbf{2 b}$ : Intraoperative finding of a skin defect after surgical excision

Figure: 2c: Postoperative finding

The topic of drug-induced oncologic diseases is becoming more popular in the scientific community $[5,8]$. Especially growing attention draws the group of the angiotensin receptor blockers, which seems to be possibly associated with increased risk of oncologic diseases [4-6, 8-10]. The prolonged antihypertensive therapy with sartans (ARB's), but also with thiazide diuretics could be considered as a probable key factor for developing cutaneous melanomas and other types of cancer $[4-6,8,9,11]$.

A study of Olchewski et al. (2018), the only experimental one in the subject for the moment, contains some disturbing data. According to this study, blocking angiotensin receptors inhibits melanocytes' migrational activity, but also increases their adhesion and invasion [7].

The curious in the presented case is the detailed, chronological data, which accurately describes the possible time relations between medication onset and developing neoplasms. The patient started his antihypertensive therapy with Valsatan\ Hydrochlorothiazide in 2013. Until 2015 during annual dermatological examinations, clinically and dermatoscopic suspicious was not found (BCC of the back- appearance firstly 2 years after the medication start (2015).

According to the anamnestic data the dysplastic nevus appeared for the first time in 2016 (approximately 2,5 years after the antihypertensive therapystarted). This timespan 'suspiciously' matches or is very close to other documented cases of malignant pigmented lesions after therapy with sartans $[4,8,10]$. In this case the BCC but also the pigmented malignant lesion developed after a combined therapy Valsartan/Hydrochlorothiazide. There are evidences that both medicaments increase the risk of developing not only melanoma, but also prostate carcinoma $[6,8,11]$. In our opinion the described case is the first case in medical literature of a dysplastic nevus, developed roughly 2,5 years after onset of combined antihypertensive therapy, where the cancerogenesis might have been triggered by cancerogenic adultearnts or the active substance itself.

Because of inability for a complete clarification of the possible pathogenetic connection between systemic antihypertensive drugs and developing of malignant pigmented lesions, we think that an annual dermatological check-up is highly recommended for such patients. 
The need of big, longitudinal studies in this subject is constantly increasing. They should confirm or reject our statement, because the simultaneous manifestation of two events does not necessarily mean they are causally related.

\section{References}

1. Rezze GG, Leon A, Duprat J. Dysplastic nevus (atypical nevus). Anais brasileiros de dermatologia. 2010; 85(6):863-871.doi:10.1590/ S0365-05962010000600013.

2. Pampena R, Kyrgidis A, Lallas A, Moscarella E, Argenziano G, Longo C. A meta-analysis of nevus-associated melanoma: Prevalence and practical implications. Journal of the American Academy of Dermatology. 2017,1; 77(5):938-945.doi: 10.1016/j.jaad.2017.06.149

3. Goldstein, Alisa M and Margaret A. Tucker. "Dysplastic nevi and melanoma." 2013:528-532. doi:10.1158/1055-9965.EPI-12-1346

4. Tchernev G and Temelkova I. Drug-Induced Melanoma: Irbesartan Induced Cutaneous Melanoma! First Description in the World Literature! Open access Macedonian journal of medical sciences. 2019,15; 7(1):114.doi: 10.3889/oamjms.2019.042

5. Tchernev G, Temelkova I. Additional 4 cases of valsartan/irbesartaninduced melanomas?. Journal of biological regulators and homeostatic agents. 2019, 29;33(3).:911-912.

6. Tchernev G, Temelkova I. Valsartan/hydrochlorothiazide induced prostate carcinoma in a patient who subsequently developed melanoma. Journal of biological regulators and homeostatic agents. 2019, 13; 33(4).
7. Olschewski DN, Hofschröer V, Nielsen N, Seidler DG, Schwab A, Stock C. The angiotensin II type 1 receptor antagonist losartan affects NHE1-dependent melanoma cell behavior. Cellular Physiology and Biochemistry. 2018; 45(6):2560-2576.doi:10.1159/000488274

8. Tchernev G, Temelkova I. Valsartan Induced Melanoma?! First Description in Medical Literature! Open access Macedonian journal of medical sciences. 2018, 20; 6(12):2378. doi:10.3889/ oamjms.2018.517

9. Sipahi I, Debanne S, Rowland D, Simon D, Fang JC. Angiotensinreceptor blockade and risk of cancer: meta-analysis of randomised controlled trials. Lancet Oncol. 2010;11(7):627-636.doi: 10.1016/ S1470-2045(10)70106-6

10. Tchernev G and Temelkova I. Olmesartan/valsartan induced giant achromatic cutaneous melanoma:" modified" one-step surgical approach with favourable outcome. Journal of biological regulators and homeostatic agents. 2019, 20; 33(6).

11. Pottegård A, Pedersen SA, Schmidt SA, Hölmich LR, Friis S, Gaist D. Association of hydrochlorothiazide use and risk of malignant melanoma. JAMA internal medicine. 2018; 178(8): 1120-1122. doi:10.1001/jamainternmed.2018.1652 